[8] B. R. Barmish and P. Khargonekar. "Robust stability of feedback control systems with uncertain parameters and unmodelled dynamics," in Proc. 1988 Amer. Contr. Conf., vol. 3, Atlanta, GA, pp. 1857-1862

[9] R. K. Yedavalli, "Stability analysis of interval matrices-Another sufficient condition." Int. J. Contr. vol. 43, no. 3, pp. 767-772, 1985.

[10] K. H. Wei and R. K. Yedavalli, "Invariance of strict Hurwitz property for uncertain polynomials witt dependent coefficients." IEEE Trans. Automat. Contr. vol. AC-32. pp. 907-909. Oct. 1987.

\section{Pole Placement Under Structural Constraints}

\section{A. PIAZZI}

Abstract-The spectral assignability properties of the linear map $A+B F$ are exploited under the structural constraints that a given controlled invariant $\nabla$ complement of im $B$ are both $(A+B F)$-invariants. Correlations with previous results are established, and the duality is performed with conditioned invariants. Direct applications are found in the linear geometric theory of many reduced-order syntheses, e.g., those of observers, regulators, etc.

\section{INTRODUCTION}

In the current section, we set the notation and present a known result on the spectral assignability of $A+B F$. This result will be generalized in Section II by Lemma 2 , and in the last section, we report two corollaries. an application of the found result.

With the capital scripts $X, \mathcal{U}$, and $\mathcal{Y}$ we denote real vector spaces of order, respectively, $n, q$, and $p$. The direct sum of subspaces, the sum of disjointed subspaces, is designated by . We introduce the linear maps $A: \mathfrak{X} \rightarrow \mathfrak{X}, B: \mathcal{U} \rightarrow \mathfrak{X}, C: \mathfrak{X} \rightarrow \mathcal{Y}$, and define $\mathfrak{N}:=$ im $B$ as the image of $B$ and $\mathcal{C}:=\operatorname{ker} C$ as the kernel of $C$. In general, matrices and linear maps are denoted by the same symbols, relying on the context for the appropriate distinction.

A subspace $\mathfrak{I} \subseteq \mathfrak{X}$ is an $A$-invariant if $A \mathfrak{I} \subseteq \mathfrak{S} . A|\mathfrak{I} A| \mathfrak{X} / \mathfrak{I}$ are, respectively, the restriction on $\mathfrak{I}$ of $A$ and the map induced by $A$ on the quotient space $\mathfrak{X} / \mathcal{I} . \sigma(A)$ signifies the spectrum of $A$ and $\uplus$ the set union with repetition.

Controlled invariants, also known as $(A, B)$-invariants, are subspaces $\checkmark \subseteq \mathscr{X}$ such that $A \nabla \subseteq \nabla+\mathcal{Q}$ and conditioned invariants are subspaces $\mathcal{S} \subseteq \mathcal{X}$ satisfying $A(\mathcal{S} \cap \mathcal{C}) \subseteq \mathcal{S}$

Given a controlled invariant $\vee$, let $F(\mathcal{V})$ be the set of all maps $F: X \rightarrow \mathcal{U}$ such that $(A+B F) \mathcal{V} \subseteq$ V. $Q$ denotes the reachable subspace of the pair $(A, B)$ and $\mathcal{R}_{0}$ the reachable subspace constrained on ๆ. As is known, $\mathrm{R}=\langle A \mid \mathrm{B}\rangle$ (i.e., $\mathbb{B}+A\left(B+A^{2}\left(B+\cdots+A^{n-1} B\right.\right.$ ) and $R_{v}=\langle A+B F \mid \beta \cap V\rangle$ with $F \in F(\mathcal{V})(R$, does not depend on the particular chosen $F)$; hence, $R$, and $\nabla+Q$ are $(A+B F)$-invariants for any $F \in F(V)$. Schumacher [1] proved the following result.

Property 1: Given a pair $(A, B)$ and any controlled invariant $\vee$. The freedom in placing the poles of $A+B F$ when $F$ is restricted to $F(v)$ can be described as follows:

$$
\begin{gathered}
\sigma\left(A+B F \mid \mathcal{Q}_{v}\right) \text { is free } \\
\sigma\left(A+B F \mid \mathcal{V} / \mathcal{R}_{v}\right) \text { is fixed } \\
\sigma(A+B F \mid \vee+Q / \vee) \text { is free } \\
\sigma(A+B F \mid X / \vartheta+Q) \text { is fixed. }
\end{gathered}
$$

Manuscript received December 9, 1988; revised June 1. 1989.

The author is with the Department of Electronics. Computers, and Systems, University of Bologna, Viale Risorgimento 2, 40136 Bologna. Italy.

IEEE Log Number 9035076

\section{LEMMAS}

With respect to Property 1 , the further structural constraint that a complement $v_{1}$ of $B$ is to become an $(A+B F)$-invariant and $v$ is to

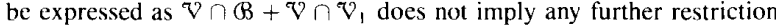
on the spectral assignability, which is indeed the same as in Property 1. This is stated in the following lemma.

Lemma 2: Given a pair $(A, B)$ and any controlled invariant $\vee$, there exist both a controlled invariant $V_{1}$ and a linear map $F: X \rightarrow \mathcal{U}$ such that

$$
\begin{gathered}
\beta \because \nabla_{1}=x \\
\vee=\vee \cap B+\vee \cap \nabla_{1} \\
(A+B F) \nabla_{1} \subseteq \nabla_{1} \\
(A+B F) \vee \subseteq \vee
\end{gathered}
$$

and with the following possibility of pole placement:

$$
\begin{gathered}
\sigma\left(A+B F \mid \mathcal{R}_{v}\right) \text { is free } \\
\sigma\left(A+B F \mid \vee / \mathcal{R}_{v}\right) \text { is fixed } \\
\sigma\left(A+\left.B F\right|^{\vee}+\mathbb{R} / \mathcal{V}\right) \text { is free } \\
\sigma(A+B F \mid \mathfrak{X} / \mathcal{V}+\mathbb{R}) \text { is fixed. }
\end{gathered}
$$

Proof: Let us perform the basis change in the state space according to

$$
x=T x^{\prime}
$$

where $T$ is a nonsingular matrix partitioned as $\left[T_{1} T_{2} T_{3} T_{4} T_{5} T_{6}\right]$ with $\operatorname{im} T_{1}=\Re \cap \mathcal{Q}, \operatorname{im}\left[T_{1} T_{2}\right]=\mathcal{R}_{1^{\prime}}, \operatorname{im}\left[T_{1} T_{2} T_{3}\right]=\mathcal{V}, \operatorname{im}\left[T_{1} T_{4}\right]=\mathscr{B}$, and $\operatorname{im}\left[T_{1} T_{2} T_{3} T_{4} T_{5}\right]=V+R$. On the basis of state variables $x^{\prime}$, the system and control matrices are expressed by

$$
\begin{aligned}
& A^{\prime}:=T^{-1} A T=\left[\begin{array}{cccccc}
A_{11}^{\prime} & A_{12}^{\prime} & A_{13}^{\prime} & A_{14}^{\prime} & A_{15}^{\prime} & A_{16}^{\prime} \\
A_{21}^{\prime} & A_{22}^{\prime} & A_{23}^{\prime} & A_{24}^{\prime} & A_{25}^{\prime} & A_{26}^{\prime} \\
0 & 0 & A_{33}^{\prime} & A_{34}^{\prime} & A_{35}^{\prime} & A_{36}^{\prime} \\
A_{41}^{\prime} & A_{42}^{\prime} & A_{43}^{\prime} & A_{44}^{\prime} & A_{45}^{\prime} & A_{46}^{\prime} \\
0 & 0 & 0 & A_{54}^{\prime} & A_{55}^{\prime} & A_{56}^{\prime} \\
0 & 0 & 0 & 0 & 0 & A_{66}^{\prime}
\end{array}\right], \\
& B^{\prime}:=T^{-1} B=\left[\begin{array}{c}
B_{1}^{\prime} \\
0 \\
0 \\
B_{4}^{\prime} \\
0 \\
0
\end{array}\right] .
\end{aligned}
$$

The structural zeros in $A^{\prime}$ are due to the $A$-invariance of $\mathcal{V}+\mathbb{R}$ and the $(A, B)$-controlled invariance of $\mathrm{R}_{v}$ and $\nabla$.

Considering matrix $B$ of full rank (without any loss of generality), we make the basis change, in the input space $\mathcal{U}$.

$$
u=N v
$$

having defined $N$ as

$$
N:=\left[\begin{array}{l}
B_{1}^{\prime} \\
B_{4}^{\prime}
\end{array}\right]^{-1} .
$$


The control matrix in the coordinates $x^{\prime}$ and $v$ is given by

$$
B^{\prime \prime}=B^{\prime} N=\left[\begin{array}{cc}
I_{1} & 0 \\
0 & 0 \\
0 & 0 \\
0 & I_{4} \\
0 & 0 \\
0 & 0
\end{array}\right]
$$

with the identity matrices $I_{1}$ and $I_{4}$ of order equal to, respectively, dim $(B \cap V)$ and $\operatorname{dim}(\mathcal{V}+Q / \mathcal{V})$.

By virtue of the properties of $\mathcal{R}_{v}$ and $\mathcal{R}$, the following pairs are controllable:

$$
\begin{aligned}
& \left(\left[\begin{array}{ll}
A_{11}^{\prime} & A_{12}^{\prime} \\
A_{21}^{\prime} & A_{22}^{\prime}
\end{array}\right],\left[\begin{array}{c}
I_{1} \\
0
\end{array}\right]\right) \\
& \left(\left[\begin{array}{cc}
A_{44}^{\prime} & A_{45}^{\prime} \\
A_{54}^{\prime} & A_{55}^{\prime}
\end{array}\right],\left[\begin{array}{c}
I_{4} \\
0
\end{array}\right]\right) .
\end{aligned}
$$

On the other hand, the controllability of pairs (4) and (5) implies, by means of the rank criterion, that pairs $\left(A_{22}^{\prime}, A_{21}^{\prime}\right)$ and $\left(A_{55}^{\prime}, A_{54}^{\prime}\right)$ are also controllable. Hence, there exist matrices $F_{12}^{\prime}$ and $F_{45}^{\prime}$ such that the spectra $\sigma\left(A_{22}^{\prime}+A_{21}^{\prime} F_{12}^{\prime}\right)$ and $\sigma\left(A_{55}^{\prime}+A_{54}^{\prime} F_{45}^{\prime}\right)$ are arbitrarily assignable.

Consider now the further basis change in the state space

$$
x^{\prime}=\tilde{T} \tilde{x}
$$

where $T^{\prime}$ is defined as

$$
\tilde{T}:=\left[\begin{array}{cccccc}
I_{1} & F_{12}^{\prime} & 0 & 0 & 0 & 0 \\
0 & I_{2} & 0 & 0 & 0 & 0 \\
0 & 0 & I_{3} & 0 & 0 & 0 \\
0 & 0 & 0 & I_{4} & F_{45}^{\prime} & 0 \\
0 & 0 & 0 & 0 & I_{5} & 0 \\
0 & 0 & 0 & 0 & 0 & I_{6}
\end{array}\right] .
$$

The system and control matrices according to coordinate transformations (3) and (6) are given by

$\tilde{A}:=\tilde{T}^{-1} A^{\prime} \tilde{T}=\left[\begin{array}{cccccc}\tilde{A}_{11} & \tilde{A}_{12} & \tilde{A}_{13} & \tilde{A}_{14} & \tilde{A}_{15} & \tilde{A}_{16} \\ A_{21}^{\prime} & A_{22}^{\prime}+A_{21}^{\prime} F_{12}^{\prime} & A_{23}^{\prime} & A_{24}^{\prime} & \tilde{A}_{25} & A_{26}^{\prime} \\ 0 & 0 & A_{33}^{\prime} & A_{34}^{\prime} & \dot{A}_{35} & A_{36}^{\prime} \\ A_{41}^{\prime} & \tilde{A}_{42} & A_{43}^{\prime} & \tilde{A}_{44} & \dot{A}_{45} & \tilde{A}_{46} \\ 0 & 0 & 0 & A_{54}^{\prime} & A_{55}^{\prime}+A_{54}^{\prime} F_{45}^{\prime} & A_{56}^{\prime} \\ 0 & 0 & 0 & 0 & 0 & A_{66}^{\prime}\end{array}\right]$

$\tilde{B}:=\tilde{T}^{-1} B^{\prime \prime}=\left[\begin{array}{cc}I_{1} & 0 \\ 0 & 0 \\ 0 & 0 \\ 0 & I_{4} \\ 0 & 0 \\ 0 & 0\end{array}\right]$.

We define in the coordinates $\tilde{x}$ and $v$ the subspace $V_{1}$ and the map $F$, and we will verify - in the same coordinates-all the claimed assertions:

$$
\begin{aligned}
& \nabla_{1}:=\operatorname{im}\left[\begin{array}{cccc}
0 & 0 & 0 & 0 \\
I_{2} & 0 & 0 & 0 \\
0 & I_{3} & 0 & 0 \\
0 & 0 & 0 & 0 \\
0 & 0 & I_{5} & 0 \\
0 & 0 & 0 & I_{6}
\end{array}\right] \\
& \tilde{F}:=\left[\begin{array}{cccccc}
-\tilde{A}_{11}+P & -\tilde{A}_{12} & -\tilde{A}_{13} & R & -\tilde{A}_{15} & -\tilde{A}_{16} \\
-A_{41}^{\prime} & -\dot{A}_{42} & -A_{43}^{\prime} & -\tilde{A}_{44}+Q & -\tilde{A}_{45} & -\tilde{A}_{46}
\end{array}\right]
\end{aligned}
$$

where $P$ and $Q$ are free matrices with arbitrary eigenvalues and matrix $R$ is a further free parameter.

The structural conditions (1a) and (lb) are verified immediately; to prove the remaining conditions, look closely at matrix $\tilde{A}+\tilde{B} \tilde{F}$ :

$$
\dot{A}+\tilde{B} \tilde{F}=\left[\begin{array}{cccccc}
P & 0 & 0 & \tilde{A}_{14}+R & 0 & 0 \\
A_{21}^{\prime} & A_{22}^{\prime}+A_{21}^{\prime} F_{12}^{\prime} & A_{23}^{\prime} & A_{24}^{\prime} & \dot{A}_{25} & A_{26}^{\prime} \\
0 & 0 & A_{33}^{\prime} & A_{34}^{\prime} & \dot{A}_{35} & A_{36}^{\prime} \\
0 & 0 & 0 & Q & 0 & 0 \\
0 & 0 & 0 & A_{54}^{\prime} & A_{55}^{\prime}+A_{54}^{\prime} F_{45}^{\prime} & A_{56}^{\prime} \\
0 & 0 & 0 & 0 & 0 & A_{66}^{\prime}
\end{array}\right] .
$$

The structural zeros of matrix (7) show the $(A+B F)$-invariance of subspaces $V_{1}$ and $V$ [inclusions $(\mathrm{lc})$ and $(1 \mathrm{~d})$ ]. The last four conditions derive from the following spectra identities:

$$
\begin{gathered}
\sigma\left(A+B F \mid \mathcal{R}_{v}\right)=\sigma(P) \uplus \sigma\left(A_{22}^{\prime}+A_{21}^{\prime} F_{12}^{\prime}\right) \\
\sigma\left(A+B F \mid \vee / \mathcal{R}_{v}\right)=\sigma\left(A_{33}^{\prime}\right) \\
\sigma\left(A+B F(\mathcal{V}+\Re / \vee)=\sigma(Q) \uplus \sigma\left(A_{55}^{\prime}+A_{54}^{\prime} F_{45}^{\prime}\right)\right. \\
\sigma(A+B F \mid \mathfrak{X} / \vee+R)=\sigma\left(A_{66}^{\prime}\right) .
\end{gathered}
$$

Remark: Note that the given proof is fully constructive and provides the map $F$ and the subspace $V_{1}$, which both depend on the chosen spectra.

Given a pair $(C, A)$ and a conditioned invariant $S$, let $\mathcal{Q}$ be the unobservable subspace of the pair $(C, A)$ and let $\mathcal{Q}$ be the unobservable subspace containing $\mathcal{S}$. Lemma 2 can be dualized-in terms of conditioned invariants - in the following way.

Lemma $2^{\prime}$ : Given a pair $(C, A)$ and any conditioned invariant $S$, there exist both a conditioned invariant $S_{1}$ and a linear map $G: \mathcal{Y} \rightarrow \mathfrak{X}$ such that

$$
\begin{gathered}
e-\mathrm{s}_{1}=x \\
\mathrm{~s}=\mathrm{s} \cap \mathrm{e}+\mathrm{s} \cap \mathrm{S}_{1} \\
(A+G C) \mathrm{s}_{1} \subseteq \mathrm{S}_{1} \\
(A+G C) \mathrm{s} \subseteq \mathrm{S}
\end{gathered}
$$

and with the following possibility of pole placement:

$$
\begin{gathered}
\sigma(A+G C \mid \mathcal{Q} \cap \S) \text { is fixed } \\
\sigma(A+G C \mid \mathrm{S} / \mathcal{Q} \cap S) \text { is free } \\
\sigma\left(A+G C \mid \mathcal{Q}_{.} / S\right) \text { is fixed } \\
\sigma\left(A+G C \mid \mathfrak{X} / \mathcal{Q}_{\Omega}\right) \text { is free. }
\end{gathered}
$$




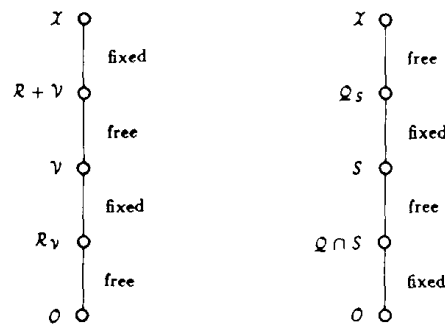

Fig. 1. Hasse diagrams of controlled and conditioned invariants.

The pole placement possibilities can be visualized by the Hasse diagrams shown in Fig. 1 where the free and fixed spectra are exhibited.

\section{Corollaries}

From Lemmas 2 and $2^{\prime}$, we can derive a variety of corollaries depending on the assumptions we choose for the specific applications. For example, the following result has been presented by Imai and Akashi for dealing with stable disturbance rejection compensators [2] (see also [3]).

Corollary 3: Let pair $(A, B)$ be controllable. Given any internally stabilizable controlled invariant $\nabla$, there exists a controlled invariant $\nabla_{1}$ such that

$$
\begin{gathered}
\boldsymbol{\beta} \oplus \nabla_{1}=x \\
\nabla=\nabla \cap \beta+\nabla \cap \nabla_{1}
\end{gathered}
$$

$\vartheta_{2}$ is internally stabilizable.

A specialization of Lemma $2^{\prime}$ leads to the geometric theory of reducedorder observers, as we shall see shortly (see [4]).

Corollary 4: Given an observable pair $(C, A)$, there exist a conditioned invariant $\&_{1}$ and a map $G$ such that

$$
\begin{gathered}
\mathcal{C}-\mathcal{S}_{1}=\mathfrak{X} \\
(A+G C) \mathcal{S}_{1} \subseteq \mathcal{S}_{1} \\
\sigma\left(A+G C \mid X / \delta_{1}\right) \text { is free. }
\end{gathered}
$$

Proof: With regard to Lemma $2^{\prime}$, we choose $S:=X$ which is, evidently, a $(\mathcal{C}, \mathfrak{Q})$-conditioned invariant. The subspace $S_{1}$ satisfies (10a) and $(10 \mathrm{~b})$. Since the unobservable subspace $Q$ is equal to $\mathcal{O}$ (the zero subspace), it follows from (8f) that $\sigma(A+G C)$ is freely assignable. But $\sigma(A+G C)=\sigma\left(A+G C \mid S_{1}\right) \oplus \sigma\left(A+G C \mid \mathfrak{X} / \mathcal{S}_{1}\right)$; therefore, we obtain condition $(10 \mathrm{c})$.

Consider the observable system

$$
\Sigma\left\{\begin{array}{l}
\dot{x}(t)=A x(t)+v(t) \\
y(t)=C x(t)
\end{array}\right.
$$

where $x, v$, and $y$ are, respectively, the state, the input, and the output vectors. Using Corollary 4 , we can immediately synthesize for system $\Sigma$ an observer with order $n-p$ of arbitrary dynamic response. Indeed, write the observer equations as

$$
\Sigma_{0}\left\{\begin{array}{l}
z(t)=Q_{1}(A+G C) T_{1} z(t)-Q_{1} G y(t)+Q_{1} v(t) \\
\hat{x}(t)=L_{1} y(t)+T_{1} z(t)
\end{array}\right.
$$

where $L_{1}, Q_{1}, T_{1}$ are full-rank matrices satisfying $L_{1} C+T_{1} Q_{1}=I$ with ker $Q_{1}=S_{1}$ and $G$ and $S_{1}$ are synthesized according to Corollary $4 ; z$ is the observer state and $\hat{x}$ is the estimate of state $x$. The block diagram of $\Sigma$ and $\Sigma_{0}$ is depicted in Fig. 2 .

It would not be difficult, and it is left to the interested reader, to prove that $\lim _{t \rightarrow \infty} \hat{x}(t)=x(t) \forall x(0), z(0)$ and the dynamic response of $\Sigma_{0}$ is given by the spectrum $\sigma(A+G C \mid X / \&)$.

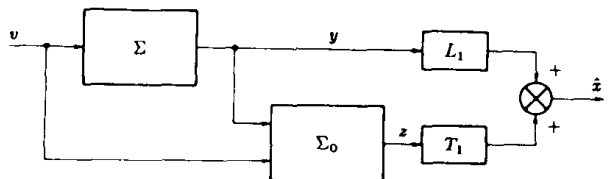

Fig. 2. State estimation through a reduced-order observer.

Perhaps a more interesting application of Lemmas 2 and $2^{\prime}$ can be found in the geometric theory of reduced-order regulators [5]-[7]. In fact, in these applications, the synthesis procedure can take advantage of the fact that the above lemmas do not need any hypothesis on either pair $(A, B)$ or $(C, A)$.

\section{References}

[1] J. M. Schumacher, "Complement on pole placement," IEEE Trans. Automat Contr., vol. AC-25, pp. 281-282, 1980.

[2] $\mathrm{H}$. Imai and $\mathrm{H}$. Akashi, "Disturbance localization and pole shifting by dynamic compensation." IEEE Trans. Automat Contr., vol. AC-26, pp. 226-235, 1981.

[3] A. Piazzi. "Geometric aspects of reduced-order compensators for disturbance rejection," submitted to IEEE Trans. Automat. Contr.

[4] W. M. Wonham, "Dynamic observers-Geometric theory," IEEE Trans. Automat. Contr., vol. AC-15, pp. 258-259, 1970.

15i A. Piazzi, "Una nuova impostazione geometrica di alcuni problemi della teoria dei sistemi lineari," Ph.D. dissertation. Dep. Electron.. Comput., Syst.. Univ. Bologna, Bologna, Italy, Dec. 1986.

[6] G. Marro and A. Piazzi, "Duality of reduced-order regulators," in Proc. Int. AMSE Conf., Modelling \& Simulation, vol. 1B, Istanbul, Turkey, June 1988. pp. 113-121.

[7] G. Marro, in Teoria dei Sistemi e del Controllo, X. Zanichelli, Ed. Bologna, Italy. 1989 , ch. 7. pp. 259-267.

\section{Comments on "Adaptive Manipulator Control: A Case Study"}

\section{MARK W. SPONG, ROMEO ORTEGA, AND RAFAEL KELLY}

\section{Abstract - Stability in the sense of Lyapunov of the adaptive motion controller for robot manipulators reported in the paper ${ }^{1}$ is established} in this note.

It is well known (see, e.g., [1]) that to carry out the stability analysis of most adaptive systems, the well-known Lyapunov techniques cannot be directly applied. Instead, a problem specific mathematical machinery has been developed which, roughly speaking, proceeds as follows. First, a nonnegative function of (not necessarily all) the states of the system is used to establish boundedness and square integrability of some signals of interest. Then, chasing the signals through the loop, global boundedness of all signals and asymptotic error tracking is proven. It is worth underscoring that, in general, this procedure does not allow us to establish stability in the sense of Lyapunov. This situation may cast doubts on the robustness properties of the adaptive systems. See [2], and references therein, for further details.

This same procedure for stability analysis is used in the paper ${ }^{\prime}$ for an adaptive motion controller for robot manipulators. The proposed con-

Manuscript received February 14, 1989

M. W. Spong is with the Coordinated Science Laboratory. University of Illinois, Urbana-Campaign, IL 61801.

R. Ortega and R. Kelly are with DEPFI, Universidad Nacional Autonoma de Mexico, Mexico.

IEEE Log Number 9034504

'J. Slotine and W. Li, IEEE Trans. Automat. Contr., vol. 33, pp. 995-1003, Nov. 